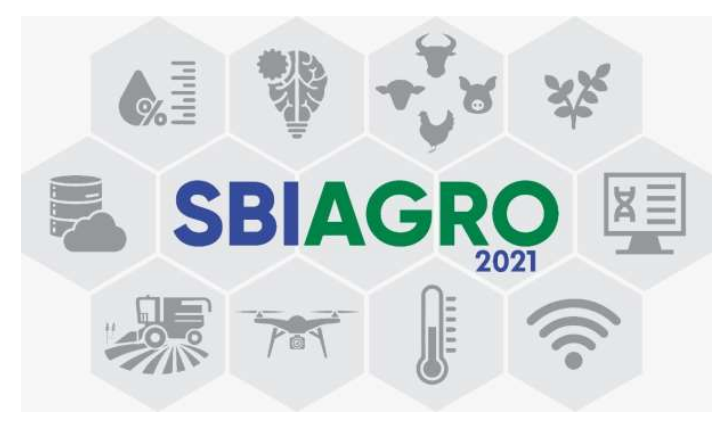

\title{
Simulação de Sistemas de Produção de Novilho Precoce: uma Aplicação Prática
}

\author{
Roberto A. F. Abreu ${ }^{1}$, Thiago Basso ${ }^{2}$, Camilo Carromeu ${ }^{3}$, Rodrigo da Costa \\ Gomes $^{3}$, Fernando R. T. Dias ${ }^{3}$, Débora Maria Barroso Paiva ${ }^{1}$
}
${ }^{1}$ Faculdade de Computação - Universidade Federal de Mato Grosso do Sul (UFMS) - Campo Grande, MS - Brasil
${ }^{2}$ Universidade Federal da Grande Dourados (UFGD) - Dourados, MS - Brasil
${ }^{3}$ Embrapa Gado de Corte - Campo Grande, MS - Brasil
abreu.roberto@ufms.br, thiagobasso@ufgd.edu.br,
camilo.carromeudembrapa.br, rcostagomes@gmail.com,
fernando.dias@embrapa.br, debora.paiva@ufms.br

\begin{abstract}
The development of software to support the sciences as a new platform for scientific research and experimentation has generated contributions to several areas of knowledge and is considered a trend for the coming years. In this article, the +Precoce platform is presented, focused on the field of Livestock, which offers models of beef cattle production systems and allows rural producers to make simulations considering their particular data. The results obtained show that the platform can contribute to decision making in rural areas and generate several benefits, such as improving the quality of brazilian meat.
\end{abstract}

Resumo. $O$ desenvolvimento de software para apoiar as ciências como uma nova plataforma de pesquisa e experimentação científica tem gerado contribuições para diversas áreas de conhecimento e é considerada uma tendência para os próximos anos. Neste artigo apresenta-se a plataforma +Precoce, voltada para o domínio da Pecuária, que oferece modelos de sistemas de produção de gado de corte e permite que produtores rurais façam simulações considerando seus dados particulares. Os resultados obtidos mostram que a plataforma pode contribuir para a tomada de decisão no meio rural e gerar diversos beneficios, tais como a melhoraria da qualidade da carne brasileira. 


\section{Introdução}

O setor de agronegócio possui papel importante para o desenvolvimento da economia e sociedade brasileira. O notável crescimento do agropecuário brasileiro tem se sustentado no aumento da produtividade, muito mais do que na ocupação de novas terras, pela aplicação de tecnologias e conhecimento acumulado tanto pelos produtores quanto por instituições que integram as cadeias produtivas do agronegócio. A inovação é a base para o desenvolvimento sustentável e atributo potencializador para criação de novas oportunidades e enfrentamento de desafios impostos por mercados globalizados (Santos et al., 2011).

O crescente uso de diferentes tecnologias envolvendo, por exemplo, biotecnologia e tecnologia da informação tem elevado o potencial de criação de produtos e processos disruptivos e de alto impacto para o setor de agropecuária. Considerando ainda a intensificação do mercado de agricultura e pecuária digital, tanto os avanços da biologia sintética e das novas tecnologias aplicadas a sistemas genéticos complexos, quanto a expansão da bioinformática na análise e no compartilhamento de dados científicos são caminhos que tendem a se intensificar (Embrapa Visão 2030). Considerando estas tendências e o volume de dados que pode ser gerado, surge a necessidade de prover apoio computacional à experimentação científica (e-Ciência ou eScience) no sentido de auxiliar pesquisadores a explorar coleções de dados. Estas novas demandas certamente impõem grandes desafios que precisam ser enfrentados, considerando a modelagem, o gerenciamento e a apresentação de dados.

A plataforma +Precoce (Abreu, 2020, Basso, 2018, Basso et al., 2018) é um projeto desenvolvido em parceria entre a Embrapa Gado de Corte, Embrapa Pantanal e a Faculdade de Computação da Universidade Federal de Mato Grosso do Sul (UFMS). O projeto recebe financiamento da Embrapa e está inserido no contexto do "Precoce MS", programa responsável pelo incentivo à produção de bovinos precoces no estado de Mato Grosso do Sul, vinculado à Secretaria de Meio Ambiente, Desenvolvimento Econômico, Produção e Agricultura Familiar do governo do estado. A Plataforma +Precoce provê acesso aos técnicos rurais e produtores de gado de corte aos sistemas melhorados de produção pesquisados pela Embrapa. Utilizando um simulador o produtor pode realizar mudanças em parâmetros visando reproduzir as características de sua propriedade rural e simular estes sistemas de produção.

De forma geral, a plataforma + Precoce considera sistemas de produção de gado de corte e simplifica sua conversão para modelos possíveis de serem simulados computacionalmente. O objetivo deste artigo é apresentar a evolução da plataforma + Precoce, em especial enfatizando seu modelo de simulação voltado para o domínio de pecuária e de apresentação de dados.

Este artigo está apresentado da seguinte forma: a Seção 2 apresenta os principais conceitos e trabalhos relacionados, a Seção 3 apresenta os principais módulos da plataforma +Precoce e a Seção 4 apresenta as considerações finais. 


\section{Background e Trabalhos Relacionados}

A pecuária de precisão é a área que estuda o uso das tecnologias de informação e comunicação na produção de bovinos. Nesta área, a tecnologia da informação é utilizada para garantir o uso de boas práticas no processo de produção pecuário almejando, assim, impulsionar a produtividade. A utilização de tecnologias pode ser feita em vários níveis e em diversas áreas da indústria bovina, lançando novos desafios para as disciplinas de Ciência da Computação no que tange o desenvolvimento de novas ferramentas e dispositivos (software e hardware) que aumentem a competitividade de estabelecimentos pertencentes à indústria pecuária (Cáceres et al., 2011).

A concorrência global impõe forte ritmo e dinamicidade na produção de gado de corte. Neste sentido, a Embrapa Gado de Corte conduz estudos há décadas visando à construção de modelos de sistemas melhorados de produção que sirvam de referência ao produtor rural. Estes modelos indicam técnicas empregadas na produção, coeficientes técnicos estimados e indicadores de desempenho econômico.

No entanto, esses modelos de sistemas geralmente estão disponibilizados no formato de publicações técnico-científicas e os indicadores são elaborados utilizando planilhas eletrônicas disponíveis apenas para pesquisadores. Essa característica dificulta o alcance e utilização pelos produtores rurais, além de dificultar o compartilhamento de atualizações e criação de novos modelos. Neste contexto, ferramentas de software têm papel cada vez mais importante uma vez que, dado o volume de informações e quantidade de variáveis que existem atualmente, é impossível tomar decisões rápidas e seguras sem o uso de ferramentas robustas.

Existem alguns simuladores disponíveis no setor agropecuário, sendo mencionados três deles a seguir:

- APSIM: simulador de sistemas agrícolas, contém módulos que permitem simulações envolvendo solo, plantas, animais e clima (APSIM Initiative, 2021);

- PaSim: provê simulações dos impactos das alterações climáticas nas pastagens e fornece feedback sobre emissão de gases do efeito estufa na atmosfera. É composto por submodelos para plantas, animais, microclima, biologia e física do solo e gerenciamento (PASIM, 2021);

- Embrapa Invernada: fornece apoio para o planejamento da produção de bovinos de corte. Permite realizar simulações do crescimento de pastagens, pastejo e crescimento de animais, com possibilidade de formular dietas otimizadas e comparar e analisar cenários de produção (Invernada, 2021).

A plataforma +Precoce apresenta como diferencial o fato de ser uma ferramenta Web para a organização e disponibilização de informações a respeito de cria, recria e engorda para a produção de bovinos destinados a programas e parcerias que bonificam carcaças por qualidade. Chamada de plataforma +Precoce em alusão ao "novilho precoce", um termo relacionado à qualidade, permite a geração de indicadores econômicos e ambientais para auxiliar o produtor na tomada de decisão e caracteriza a adequação esperada para os diversos programas e parcerias de carne de qualidade dos animais produzidos em cada sistema disponibilizado. 


\section{Plataforma +Precoce}

A plataforma é composta por quatro módulos: o Módulo Desenhista, o Módulo Simulador, o Módulo Administrador e o Módulo Portal. De forma geral, o Módulo Desenhista permite aos técnicos da Embrapa cadastrar e manter os modelos computacionais dos sistemas de produção; o Módulo Simulador é responsável pela execução das simulações dos modelos computacionais dos sistemas de produção; o Módulo Administrador gerencia as funcionalidades da plataforma relativas a cadastro de usuários perfis de acesso, logs, etc. e o Módulo Portal é a interface com o usuário final (pesquisadores, produtores e técnicos rurais).

A plataforma +Precoce permite que usuários façam a simulação e o acesso às informações de sistemas melhorados de produção de gado de corte, construídos a partir do conhecimento acumulado de experimentos da Embrapa Gado de Corte e parceiros. A aplicação deve ser flexível o suficiente para que seus usuários possam simular tais sistemas com dados o mais próximo possível de suas propriedades, de modo a colaborar com a produção de bovinos e contribuir para melhorar a qualidade da carne brasileira.

\subsection{Módulo Desenhista}

A plataforma +Precoce suporta diversos modelos de sistemas de produção de gado de corte e simplifica sua conversão para modelos passíveis de serem simulados computacionalmente. A gerência para estes modelos é propiciada pelo Módulo Desenhista, em que o ator denominado "Desenhista", ficará responsável por modelar e gerenciar os sistemas de produção considerando o modelo computacional. Este papel é exercido por pesquisadores instruídos para esta função.

Um modelo de sistema de produção é uma caixa preta em que recursos entram por suas fronteiras, são processados e geram como saídas coprodutos, resíduos ou emissões e um produto. Possuem estações e fluxos de entrada e saída. Os fluxos carregam recursos, organizados em hierarquias de tipos. Estações utilizam fórmulas de duração e fluxos utilizam fórmulas de coeficientes que envolvem parâmetros. Os valores desses parâmetros são informados pelo usuário na hora de solicitar uma simulação. A execução da simulação calcula as quantidades e datas dos fluxos e, a partir destes, os valores dos indicadores de desempenho.

Um usuário desenhista pode cadastrar, editar e excluir modelos, parâmetros e suas categorias e hierarquias, fórmulas de coeficiente, duração, valor inicial, valor residual ou vida útil, parâmetros associados ao sistema, definir os valores mínimo e máximo aceitáveis e o valor padrão. O Módulo Desenhista é responsável por gerenciar o modelo computacional permitindo que o usuário desenhista consiga inserir, ler, editar e excluir dados das entidades do modelo computacional. Este módulo foi construído utilizando o Titan Framework (Titan Framework, 2021). Na Figura 1 é possível observar uma tela que exibe os modelos cadastrados. Ao final de cada linha da tabela são exibidas as ações de visualizar, editar, excluir e copiar modelo.

Na Figura 2 é indicado um exemplo de tela de cadastro de dados. Essa tela apresenta os campos necessários para o cadastro de um fluxo. O canto superior direito apresenta o menu de ações disponíveis. É possível salvar os dados preenchidos (ação que irá processar o form, validar dados e persistir em base, no caso de sucesso na validação ou retornar erro). 


\subsection{Módulo Simulador e Módulo Administrador}

O Módulo Simulador é responsável pela execução das simulações requisitadas e, de forma geral, este módulo faz o carregamento da base de dados do modelo computacional escolhido, a execução das rotinas de cálculo de simulação e a entrega dos resultados na forma de indicadores de desempenho.

O Módulo Simulador é responsável pela execução das simulações dos modelos computacionais dos sistemas de produção, processando as requisições de simulação. Para isso, ele carrega na memória o modelo do sistema de produção e os valores dos parâmetros escolhidos para simulação e dispara a execução das rotinas de cálculo de simulação, entregando os resultados na forma de Indicadores. Devido à limitação de espaço, o Módulo Simulador não será detalhado neste artigo. Os resultados obtidos após uma simulação são apresentados no Módulo Portal, que será apresentado na subseção 3.3. De forma semelhante, o Módulo Administrador, responsável por gerenciar as funcionalidades da plataforma relativas a cadastro de usuários, perfis de acesso e outros cadastros, não será apresentado em detalhes neste artigo.

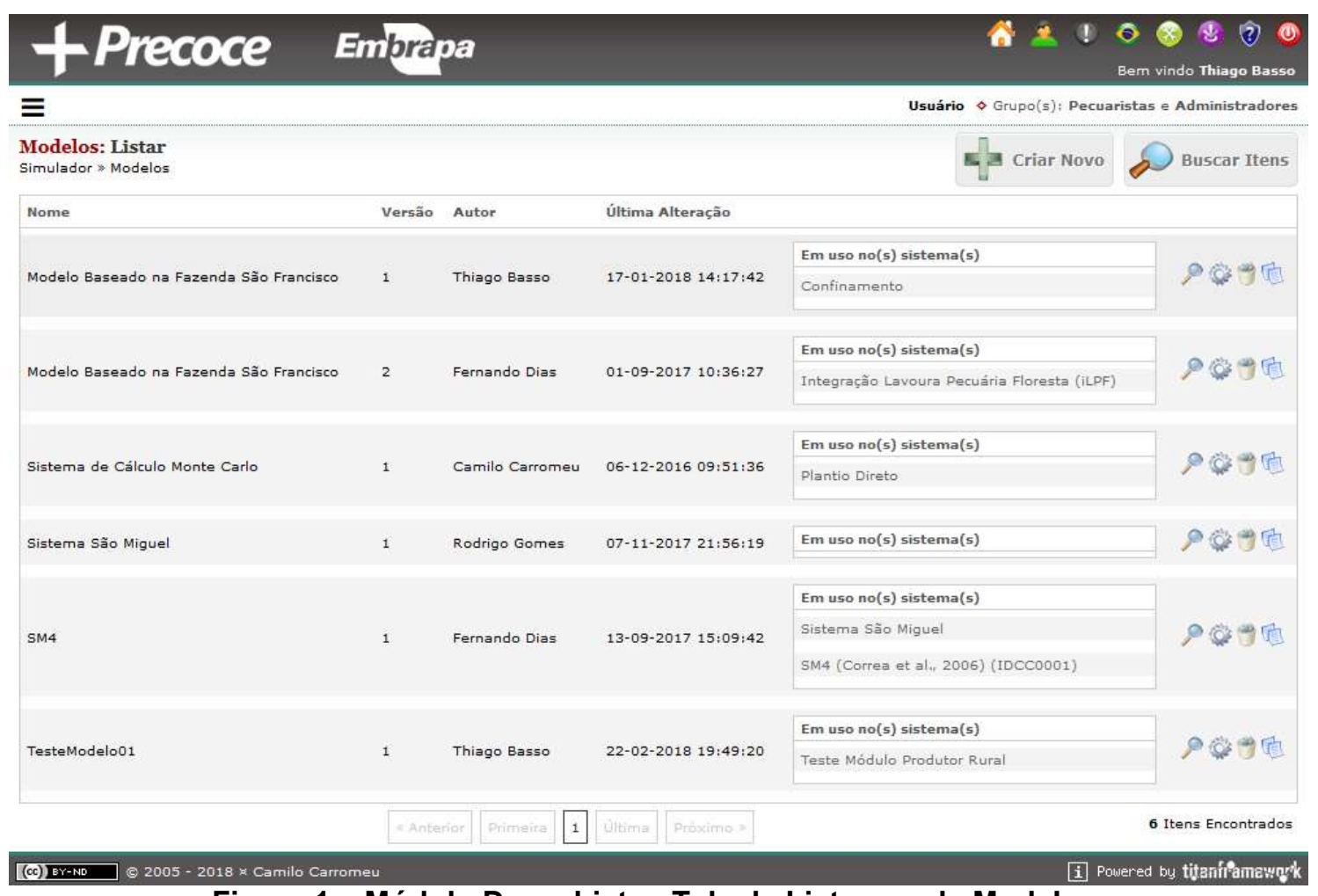

Figura 1 - Módulo Desenhista - Tela de Listagem de Modelos 


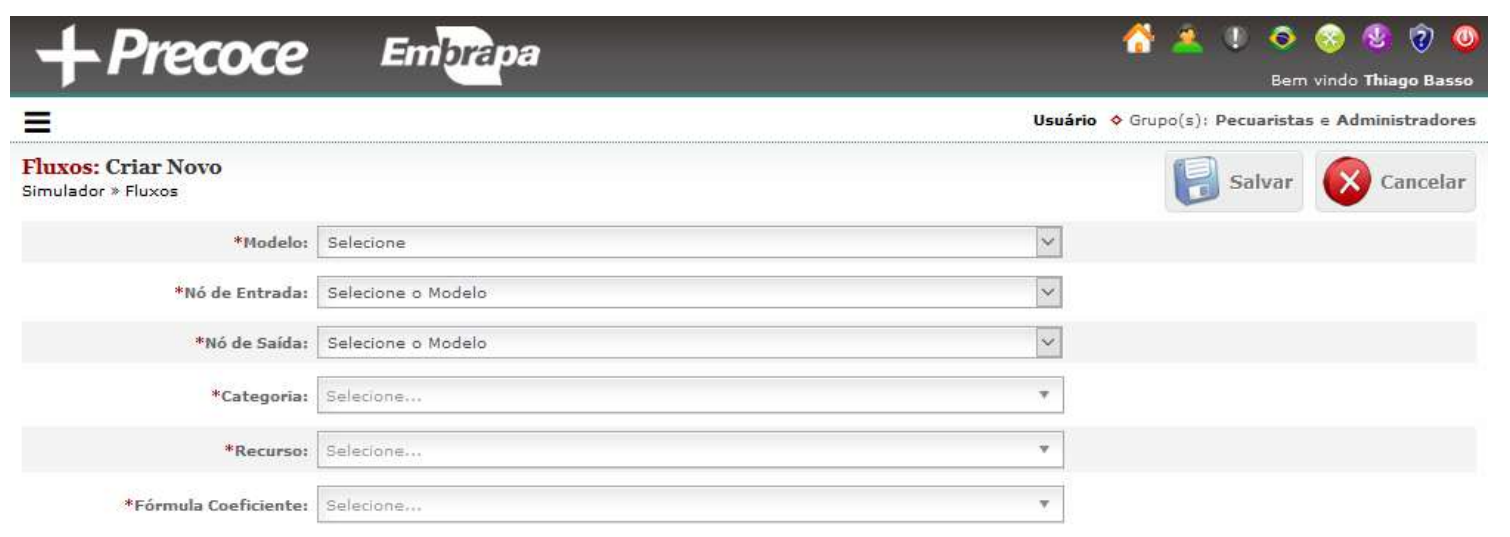

\subsection{Módulo Portal}

O Módulo Portal é a interface com o usuário final (pesquisadores, produtores e técnicos rurais). Neste módulo acontece a escolha do modelo para simulação, consulta à descrição e protocolo dos modelos de produção, consulta a simulações já realizadas, exclusão, edição ou cópia de simulações realizadas, ajuste de parâmetros das simulações e exibição dos resultados de até três simulações para comparação.

Foi utilizada a teoria de Gráfico de Gantt para representar a simulação do ciclo de reprodução de gado de corte. A representação de tarefas em barras horizontais na linha do tempo, mostra dados relevantes para tomada de decisão de forma intuitiva. Por exemplo: quando uma tarefa começa ou termina, qual tarefa se inicia antes ou depois, quais tarefas podem ser executadas em paralelo, etc. Além disso, por meio de cores é possível representar grupos de tarefas que têm algo em comum como a necessidade de um recurso ou de determinada ação.

É possível encontrar vários exemplos práticos dos benefícios do Gráfico de Gantt na literatura e que motivaram sua utilização neste trabalho. Por exemplo, Borges et al. (2013) utilizaram o Gráfico de Gantt para obter um melhor planejamento e controle da produção de uma empresa e obtiveram uma redução de 56\% no lead time, dobraram a produtividade, diminuíram os gastos com horas extras e aumentaram a satisfação do cliente.

De forma geral, o simulador da Plataforma +Precoce retorna um JSON com o resultado da simulação e o Módulo Portal executa como principais tarefas: 1) Ler o JSON; 2) Abstrair as informações necessárias para a construção de um Gráfico de Gantt que mostre as principais atividades no manejo (Cria, Recria, Monta, Engorda, etc.), em uma linha de tempo em que fique claro início e fim de cada atividade, dando uma visão cronológica de toda a cadeia servindo como um mapa para a tomada de decisões e para provisão antecipada de recursos e 3) Desenhar o gráfico na tela.

A partir do JSON retornado os dados são parseados em formato SVG (Scalabre Vectorial Graphics, criado pelo W3C - World Wide Web Consortium) para permitir a manipulação de imagens vetorizadas no navegador web. A biblioteca 3d.js (Data-Drive Documents) é utilizada para visualização dos dados. 
A Figura 3 apresenta um exemplo de uso do software desenvolvido. O usuário escolhe de uma até quatro das simulações geradas pelo simulador, se deseja comprimir as datas (traz a data das atividades para o mais cedo possível respeitando dependências) e se deseja ver as simulações escolhidas sequencialmente ou intercaladas (esta última não será apresentada neste artigo).

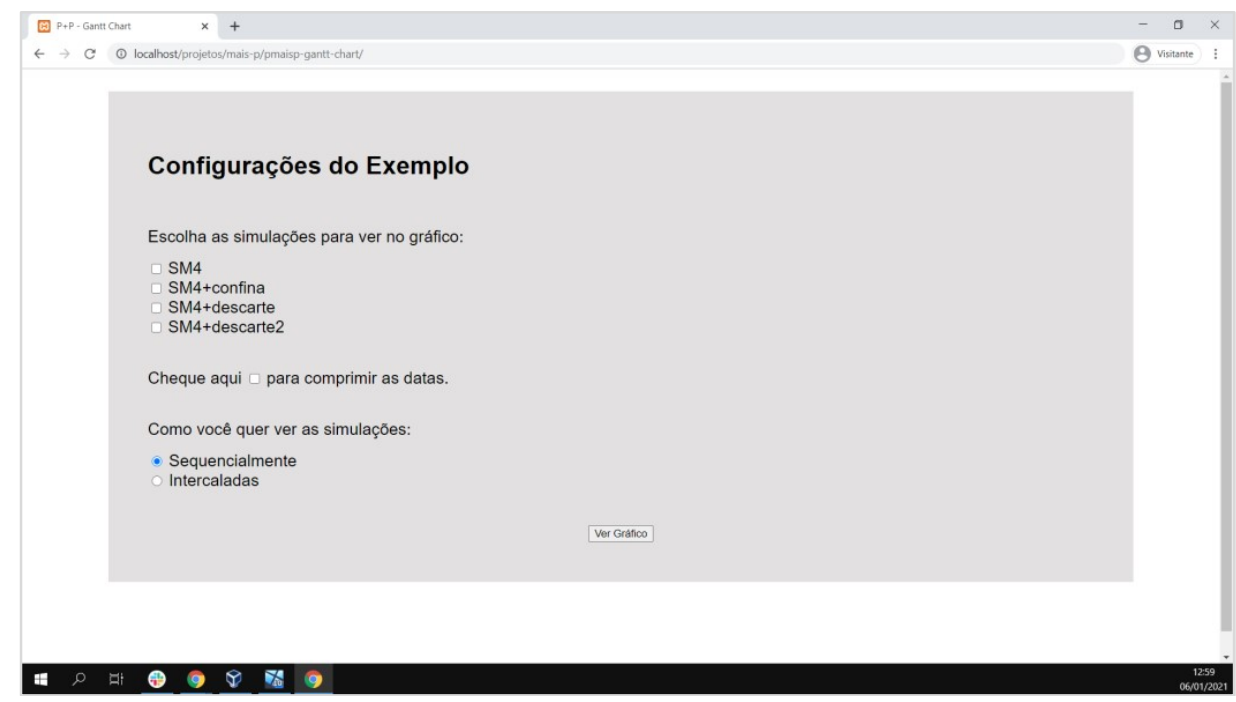

Figura 3 - Seleção de parâmetros para apresentação da simulação

Neste exemplo, o SM4 foi baseado no "Sistema Melhorado 4", descrito em https://www.infoteca.cnptia.embrapa.br/infoteca/bitstream/doc/326450/1/COT102.pdf (Corrêa et al., 2006). Os demais sistemas foram definidos apenas para testes da aplicação, e não representam sistemas reais ou recomendados pela Embrapa:

- SM4+confina: maior tempo de confinamento e menor tempo de engorda a pasto (tarefa imediatamente anterior);

- SM4+descarta: descarta (vende) todas as vacas que não emprenharam na última monta, logo após a desmama, eliminando as atividades de "reserva" destas vacas para a monta do ano seguinte;

- SM4+descarta2: semelhante ao SM4+descarta, mas vendendo também as vacas em fim de ciclo reprodutivo, que foram selecionadas para não irem para a próxima monta, com as crias, logo após o puerpério.

A Figura 4 apresenta o cronograma da simulação. Neste cronograma é apresentada a cadeia mais longa com todas as suas atividades, seguida de cadeias menores que podem ser executadas em paralelo. Ao posicionar o ponteiro do mouse sobre uma atividade, aparece uma dica (tip) informando: nome da estação, data de início e fim da atividade. Além disso, as estações são apresentadas na mesma cor facilitando a identificação dos momentos em que ocorrerá cada estação no ciclo de produção. 


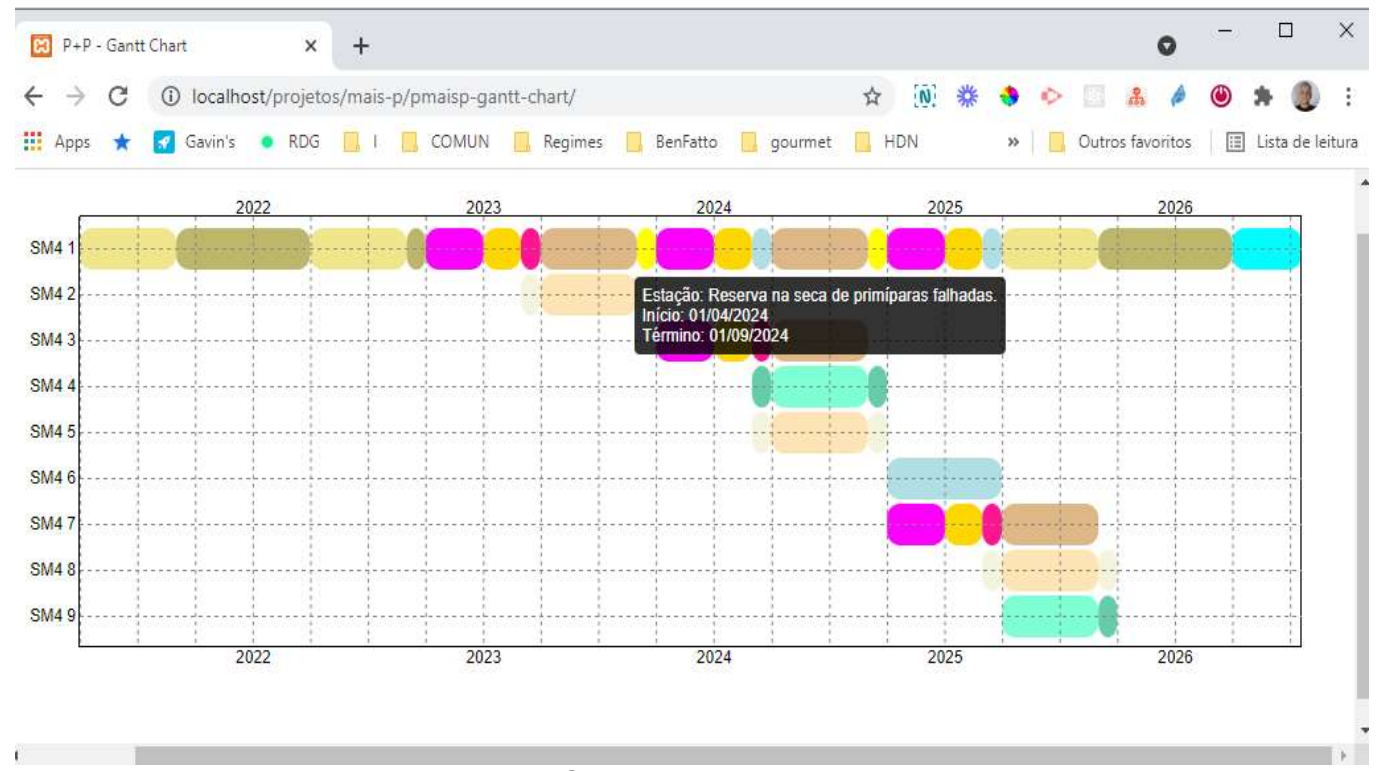

Figura 4 - Cronograma de simulação

\section{Conclusões}

A plataforma +Precoce apresenta, como principal diferencial, o fato de ser uma ferramenta computacional que promove a criação de modelos computacionais dos sistemas de produção de forma fácil, ou seja, cria um local centralizado em que o produtor rural ou pesquisadores podem criar seus próprios modelos matemáticos, cadastrar dados necessários para a simulação (de acordo com a realidade de cada usuário final) e obter informações que são apresentadas de forma simples, valorizando a usabilidade.

A principal vantagem observada é que, anteriormente ao desenvolvimento da plataforma +Precoce, os dados importantes para os produtores rurais eram disponibilizados em planilhas eletrônicas e exigiam um cuidadoso estudo e interpretação. Espera-se, a partir de agora, contribuir no sentido de prover apoio computacional à experimentação científica no domínio de pecuária. Atividades futuras estão relacionadas aos testes extensivos usando a plataforma, sobretudo, realizando estudos de caso com dados de diferentes propriedades rurais.

\section{Referências}

Abreu, R. A. F. Interface Gráfica para a Plataforma +Precoce: Uma Visão em Gantt Chart. 2020. Dissertação (Mestrado Profissional em Computação Aplicada) - Universidade Federal de Mato Grosso do Sul.

APSIM Initiative. The Agricultural Production Systems sIMulator. 2021. Disponível em: $<\mathrm{https}: / / \mathrm{www}$. apsim.info/>. Acesso em: 22.03.2021.

Basso, T.; Plataforma +Precoce: Simulador de Sistemas de Produção de Novilho Precoce. 2018. Dissertação (Mestrado Profissional em Computação Aplicada) - Universidade Federal de Mato Grosso do Sul.

Basso, T.; Gomes, R. C.; Dias, F. R. T. ; Carromeu, C. ; Cagnin, M. I. ; Paiva, D. M. B. Desenvolvimento da Plataforma +Precoce para Simulação de Sistemas de Produção de 
Novilho Precoce. In: IX Congresso Brasileiro de Software - Trilha da Indústria, 2018, São Carlos, SP.

Borges, J. P. V. et al. Planejamento e controle da produção de uma indústria de cataventos apoiado pelo gráfico de gantt: um estudo de caso. XXXIII Encontro Nacional da Engenharia de Produção. Salvador, 2013.

Cáceres, E. N. et al. Computational precision livestock-position paper. In: II Workshop of the Brazilian Institute for Web Science Research. [Rio de Janeiro, RJ: no. 02-03], 2011.

Corrêa, E. S. et al. Sistemas de produção melhorados para gado de corte em Mato Grosso do Sul. Campo Grande: Embrapa Gado de Corte, 2006. Disponível em https://www.infoteca.cnptia.embrapa.br/infoteca/bitstream/doc/326450/1/COT102.pdf (acesso em 06/01/2021).

Embrapa Visão 2030: O futuro da Agricultura Brasileira - Brasília, DF: Embrapa, 2018.

Invernada. Embrapa Invernada. 2021. Disponível em: $<$ https://www.invernada.cnptia.embrapa.br/>. Acesso em: 22.03.2021

PASIM. The Pasture Simulation Model (PaSim). 2021. Disponível em: $<$ https://www1.clermont.inra.fr/urep/modeles/pasim.htm>. Acesso em: 22.03.2021.

Santos, A. R. et al. Sw agro: Estudo do mercado brasileiro de software para o agronegócio. Estudo do mercado brasileiro de software para o agronegócio. Campinas: Embrapa Informática Agropecuária, 2011.

Titan Framewotk Titan Famework. 2021. Disponível em: <http://www.titanframework.com/>. Acesso em: 22.03.2021. 